VOL. $13(1975), 21-44$.

\title{
The regularity series of a
} \section{convergence space}

\section{G.D. Richardson and D.C. Kent}

\begin{abstract}
The regularity series, or briefly $R$-series, of a convergence space is an ordinal sequence of spaces leading to the regular modification of the space. The behavior of this series is studied relative to such basic constructs as products, subspaces, and various quotient maps. Upper bounds on the length of the $R$-series are obtained for several classes of spaces. This series can be employed to construct regular completions and compactifications.
\end{abstract}

\section{Introduction}

The definition of convergence space used here is the same as that used in [8], [9], [10], [11], and is a little more general than that introduced by Fischer [4]. This paper is a further addition to the list of papers (see references) concerned with the study of regularity in convergence spaces. The point of view taken here is, however, quite different from other papers on this subject; its nearest generic relative in the literature is a paper on the decomposition series published by the same authors (see [10]).

Fischer, [4], defined a space to be regular if, whenever $F \rightarrow x$, cl $F \rightarrow x$ ("cl" denotes the first iteration of the closure operator). Later, Cook and Fischer [3] introduced a more complicated definition of regularity which was shown by Biesterfeldt [1] to be equivalent to Fischer's original definition. Other authors have introduced other versions of regularity for convergence spaces (see, for example, [5], [7], Received 17 March 1975. 
[8], and [12]). We will use the term regular as it was originally defined by Fischer.

Following a preliminary section on notation and terminology, there is a short section stating some simple propositions about regular spaces and introducing the concept of an $R$-Hausdorff space. The next section defines the regularity series (or R-series). Later sections study the properties of this series and show how it can be applied in the study of regular compactifications and completions. A brief concluding section compares the $R$-series with the $D$-series (that is, decomposition series) which was investigated in [10].

\section{Preliminaries}

The term space will mean convergence space unless otherwise indicated. If $A \subseteq X, A \neq \emptyset$, then $A^{\bullet}$ will designate the filter consisting of all oversets of $A$; the symbol $x^{\circ}$ will be used in place of $\{x\}^{\circ}$, for $x \in X$. Given two filters $F$ and $G$ on $X$ such that $F \cap G \neq \emptyset$, for all $F \in F, G \in G$, we use $F \vee G$ to denote the filter generated by

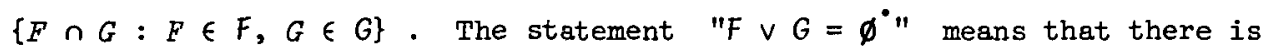
$F \in F$ and $G \in G$ such that $F \cap G=\emptyset$; the symbol $\phi^{\circ}$ does not represent a proper filter, however.

A pretopological space (called a closure space in [2] and a principa] space in [4]) is a space in which, for each point $x$, the neighborhood filter $U_{X}(x)$ at $x$ converges to $x$. (The neighborhood filter at $x$ is obtained by intersecting all filters which converge to $x$.) A pseudotopological space is characterized by the property $F \rightarrow x$ whenever each ultrafilter finer than $F$ converges to $x$.

Let $X$ be any space. The topological modification $\lambda X$ of $X$ is the finest topological space on the same underlying set coarser than $X$. The pretopological modification $\pi X$ and the pseudo-topological modification $\rho X$ are defined analagously. Note that $X, \lambda X, \pi X$, and $\rho X$ all have the same closed sets; $X, \pi X$, and $\rho X$ have the same closure operators, and $X$ and $\rho X$ have the same ultrafilter convergence.

The set of all natural numbers (including 0 ) will be denoted by $N$; $\omega$ will denote the first infinite ordinal number, and $\omega_{I}$ the first 
uncountable ordinal number. Let $X$ be a space, $A \subseteq X$, and $\alpha$ an ordinal number. The $\alpha$ th iteration of the closure of $A$ is defined recursively to be $\mathrm{cl}\left(\mathrm{cl}_{X}{ }^{\alpha-1} A\right)$ if $\alpha-1$ exists, and is defined to be $U\left\{\mathrm{cl}_{X}{ }^{\beta}: \beta<\alpha\right\}$ if $\alpha$ is a limit ordinal. For any filter $F$ on $X$, $n \in N$, we define $\mathrm{cl}_{X}{ }^{n_{F}}$ to be the filter generated by $\left\{\mathrm{cl}_{X}{ }^{n} F: F \in F\right\}$.

A space is Hausdorff if each ultrafilter converges to at most one point, and compact if each ultrafilter converges to at least one point. A space with the property that every convergent filter contains a compact set is said to be locally compact. A space is first corntable if, whenever $F \rightarrow x$, there is a filter $G$ with a countable base such that $G \rightarrow x$ and $F \geq G$.

\section{1. $R$-Hausdorff spaces}

LEMMA 1.1. Let $X$ be a space, let $F$ be an ultrafilter on $X$, and let $G$ be a filter on $X$ such that $F \geq \operatorname{cl}_{X}^{n_{G}}$. Then there is an ultrafizter $H \geq G$ such that $F \geq \operatorname{cl}_{X}{ }^{n}$.

Proof. Let $Z=\left\{K: K\right.$ a filter on $X, F \geq c^{2}{ }_{X}{ }^{n}$, and $\left.K \geq G\right\}$; let $Z$ be partially ordered by set inclusion. By Zorn's Lerma, $Z$ contains a maximal element $H$, and a straightforward argument establishes that $H$ is an ultrafilter. //

PROPOSITION 1.2. Let $X$ be a space with the property that, whenever $F$ is an ultrafilter and $F \rightarrow x$, then $\mathrm{cl}_{X} F \rightarrow x$. Then $\rho X$ is regular. In particular, $\rho X$ is regular whenever $X$ is regular.

Proof. Let $F \rightarrow x$ in $\rho X$. If ${ }^{c l} \rho X$ fails to converge to $x$ in $\rho X$, then there is an ultrafilter $G \geq c_{\rho X} F$ which fails to converge to $x$ in $X$. Since $\mathrm{cl}_{X}=c l_{\rho X}$, there exists, by Lema 1.1, an ultrafilter $H \geq F$ such that $G \geq c]_{X}$. But $H \rightarrow x$ in $X$, and so $c_{X^{H}} \rightarrow x$ in $X$ by assumption. Thus $G \rightarrow x$ in $X$, a contradiction. //

PROPOSITION 1.3. If $\left\{X_{\alpha}\right\}$ is a set of regular spaces on the same 
underlying set $X$, and $x_{0}=\sup \left\{x_{\alpha}\right\}$, then $x_{0}$ is regular.

Proof. If $F \rightarrow x$ in $X_{0}$, then $F \rightarrow x$ in $X_{\alpha}$ for all $\alpha$. Consequently, $c l_{X_{\alpha}} F \rightarrow x$ in $X_{\alpha}$ for all $\alpha$, and, since $c^{c l_{X}} F \geq c l_{X_{\alpha}} F$ for all $\alpha, \mathrm{cl}_{X_{0}} F \rightarrow x$ in $X_{0} \cdot \quad / /$

From Proposition 1.3, it follows immediately that, for any space $X$, there is a finest regular space $X_{r}$ on the same underlying set which is coarser than $X ; X_{p}$ is the supremum of all regular spaces coarser than $X$ and will be called the regular modification of $X$. A space $X$ is defined to be $R$-Hausdorff iff $X_{p}$ is Hausdorff.

In [8], a space $X$ is defined to be $T_{2}$ if distinct points $x$ and $y$ have disjoint neighborhoods (that is, if $u_{X}(x) \vee u_{X}(y)=\emptyset^{\bullet}$ ).

PROPOSITION 1.4. A compact R-Hausdorff space is $T_{2}$.

Proof. Suppose there is an ultrafilter $F \geq u_{X}(x) \vee u_{X}(y)$. Since $X$ is compact, there is $z \in X$ such that $F \rightarrow z$. Thus $\mathrm{cl}_{X} F \rightarrow z$ in $X_{p}$. But $F \geq U_{X}(x) \Rightarrow x^{\bullet} \geq{ }^{c 1}{ }_{X} F$, and so $x^{\bullet} \rightarrow z$ and $y^{\bullet} \rightarrow z$ in $X_{p}$. Since $X$ is assumed to be Hausdorff, $x=y=z$, and $X$ is $T_{2} \cdot / /$

In the following example, we show that a locally compact, first countable, $R$-Hausdorff space can fail to be $T_{2}$.

EXAMPLE 1.5. Let $X=[0,1]$, and let $X$ be equipped with the finest convergence structure subject to the following conditions:

(1) each free sequence that converges in the usual topology to $1 / n$, for some positive integer $n$, converges to 1 in $X$;

(2) each free sequence which converges to 0 in the usual topology and contains at most finitely many terms from the set $\{1 / n: n=1,2, \ldots\}$ converges to 0 in $X$.

Since convergence is defined entirely in terms of sequences and $X$ is clearly Hausdorff, it follows that $X$ is regular, $R$-Hausdorff, locally 
compact, and first countable. But $X$ is not $T_{2}$, since 0 and 1 do not have disjoint neighborhoods. //

A compact $T_{2}$ space which is not $R$-Hausdorff is constructed in Example 2.7.

\section{The $R$-series}

Let $X$ be a space. An ordinal family $\left\{r_{\alpha} X\right\}$ is defined recursively on the same underlying set.

$$
\begin{aligned}
& r_{0} X=X \text {, } \\
& F \rightarrow x \text { in } r_{1} X \text { iff there exist } n \in N \text { and } G \rightarrow x \text { in } X \text { such that } \\
& F \geq \mathrm{cl}_{X}^{n_{G}} \\
& F \rightarrow x \text { in } r_{2} X \text { iff there exist } n \in N \text { and } G \rightarrow x \text { in } X \text { such that } \\
& F \geq \mathrm{cl}_{r_{I} X}{ }^{n} G \\
& F \rightarrow x \text { in } r_{\alpha} X \text { iff there exist } n \in N, G \rightarrow x \text { in } X \text {, and } \beta<\alpha \\
& \text { such that } F \geq{ }^{c 1_{r_{B} X}}{ }^{n_{G}} \text {. }
\end{aligned}
$$

The family $\left\{r_{\alpha} X\right\}$ will be called the R-series (or regularity series) of $X$. The smallest ordinal $\gamma$ such that ${ }^{r} \gamma_{\gamma}=r_{\gamma+1} X$ is called the length of the R-series and denoted by $\tau_{R} X$.

PROPOSITION 2.1. Let $X$ be a space, $\alpha$ an ordinal number such that $z_{R} X \geq \alpha$. Then $r_{\alpha} X=X_{r}$.

Proof. Let $F \rightarrow x$ in $r_{\alpha} X$. Then $F \geq{ }^{c l_{r_{\beta}}{ }^{n} G}$ for some $\beta<\alpha$, $n \in N$, and $G \rightarrow x$ in $X \cdot{ }^{c l_{r_{\alpha}} X}{ }^{F} \geq{ }^{c l_{r_{\alpha}}} X^{c l_{r_{\beta}} X}{ }^{n}{ }^{n} \geq{ }^{c l_{r_{\alpha}} X}{ }^{n+1} G$, and so ${ }^{c r_{\alpha} X}{ }^{F} \rightarrow x$ in $r_{\alpha+1} X$. But $r_{\alpha} X=r_{\alpha+1} X$ by assumption; thus $r_{\alpha} X$ is 
regular. Since $X_{r}$ is, by definition, the finest regular space coarser than $X, \quad r_{\alpha} X \leq X_{r}$. On the other hand, it is clear from the construction of the $R$-series that $r_{\beta} X \geq X_{r}$ for each ordinal $\beta$, and thus $X_{p}=r_{\alpha} X$

PROPOSITION 2.2. If ${ }^{\mathrm{cl}}{ }_{r_{\alpha}} X^{A}={ }^{\mathrm{c}}{ }_{r_{\alpha+1}} X^{A}$ for all $A \subseteq X$, then $r_{\alpha+1} X=r_{\alpha+2} X$, and $z_{R} X \leq \alpha+1$.

Proof. Let $F \rightarrow x$ in ${ }^{r}{ }_{\alpha+2} X$. Then $F \geq c_{r_{\alpha+1}}{ }^{n_{G}}$, where $n \in N$ and $G \rightarrow x$ in $X$. By assumption, $F \geq c_{r_{\alpha} X}{ }^{n_{G}}$, and so $F \rightarrow x$ in $r_{\alpha+1} X \cdot \quad / /$

COROLLARY 2.3. If $X$ is a space such that $\pi X$ is regular, then $\tau_{R} X \leq 1$.

Proof. This follows immediately from Proposition 2.2 and the fact that $X$ and $\pi X$ have the same closure operator. //

PROPOSITION 2.4. If $X$ and $Y$ are spaces on the same underlying set with the same ultrafilter convergence, then $r_{\alpha} X$ and $r_{\alpha} Y$ have the same ultrafilter convergence for all ordinals $\alpha$, and the difference between the lengths of their R-series cannot exceed 1 .

Proof. Let $F \rightarrow x$ in $r_{1} X$, where $F$ is an ultrafilter. Then there are $n \in N$ and $G \rightarrow x$ in $X$ such that $F \geq \mathrm{cl}_{X}^{n_{G}}$. By Lemma 1.1, $F \geq \operatorname{cl}_{X} n_{H}$, where $H$ is an ultrafilter and $H \rightarrow x$ in $X$. By assumption, $H \rightarrow x$ in $Y$, and $\mathrm{cl}_{X}{ }^{n_{H}}=\mathrm{cl}_{Y}{ }^{n_{H}}$, since $X$ and $Y$ have the same ultraconvergence. Thus $F \rightarrow x$ in $r_{1} Y$. By reciprocity, $r_{1} X$ and $r_{1} Y$ have the same ultrafilter convergence. This reasoning extends by induction to all ordinals $\alpha$. The last assertion follows easily with the help of Proposition 2.2. //

Certain complications in the study of convergence spaces stem from the fact that a compact Hausdorff space can fail to be regular. The results 
that follow show that a locally compact $R$-Hausdorff space is "nearly" regular, whereas a compact Hausdorff space which is not $R$-Hausdorff can be highly non-regular.

THEOREM 2.5. If $X$ is Zocally compact and R-Hausdorff, then $\imath_{R} X \leq 1$

Proof. By Proposition 2.2, it suffices to show that ${ }^{c l_{p_{1} X}}=\mathrm{cl}_{X}$. Let $x \in \mathrm{cl}_{r_{I}} X^{A}$. Then there is an ultrafilter $F$ such that $A \in F$ and $\mathrm{F} \geq \mathrm{cl}_{X}{ }^{n_{G}}$, where $G \rightarrow x$ in $X$. By assumption, $G$ contains an $X$-closed and compact set $B$. Let $K$ be an ultrafilter which contains the filter base $\left\{A \cap \mathrm{cl}_{X}{ }^{n} G: G \in G\right\}$. Since $B \in K, K$ must converge in $X$. But $K \geq \operatorname{cl}_{X}{ }^{n} G$, and so $K \rightarrow x$ in $r_{1} X$. Since $r_{1} X$ is Hausdorfe, $K \rightarrow x$ in $X$, and $x \in \mathrm{cl}_{X} A$. //

In [8], a space is defined to be Urysohn if $X$ is Hausdorff, and, whenever $F \rightarrow x$ and $G \rightarrow y$ in $X$, with $x \neq y$, then $\left(\mathrm{cl}_{X}{ }^{n_{F}}\right) \vee\left(\mathrm{cl}_{X}{ }^{n_{G}}\right)=\phi^{\circ}$. Note that $X$ is Urysohn iff $r_{I} X$ is Hausdorff. The next observation follows immediately from this observation and Theorem 2.3 .

PROPOSITION 2.6. Each R-Hausdorff space is Umysohn. A locally compact space is Urysohn iff it is R-Hausdorff.

An example follows of a compact $T_{2}$ space with an $R$-series of length at least 3 .

EXAMPLE 2.7. For each $k \in N$, let $s_{k}=\left\{x_{i j k}: i \in N, j \in N\right\}$. We assume that each $S_{k}$ is well-ordered so as to have the order type of $N$ (although the method of well-ordering is irrelevant), and that $x_{i j k}=x_{i m n}$ iff $i=1, j=m$, and $k=n$. Let $x, y, z$ be three points not in any of the $s_{k}{ }^{\prime} s$, and let $X=\left(\cup\left\{s_{k}: k \in N\right\}\right) \cup\{x, y, z\}$. For convenience, define $A_{j k}=\left\{x_{i j k}: i \in N\right\} ; A_{j k}$ can be visualized as the $j$ th row of the infinite square matrix $s_{k}$. 
The convergence structure for $X$ is defined to be the finest subject to the following conditions:

(1) each free ultrafilter containing $A_{j k}$ converges to the $j$ th term in the well ordering of $S_{k+1}$;

(2) each free ultrafilter containing $S_{0}$, but not containing $A_{j 0}$ for any $j$, converges to $x$;

(3) each free ultrafilter containing $S_{k}$, for $k \geq 1$, but not containing $A_{j k}$ for any $j$, converges to $y$;

(4) each free ultrafilter containing $U\left\{S_{k}: k \in N\right\}$, but not containing $S_{k}$ for any $k$, converges to $z$.

From this construction, it follows that $X$ is compact and $T_{2}$. We will outline a proof that $\imath_{R^{X}} \leq 3$.

Let $F$ be a free ultrafilter on $S_{i}$ for some $i \in N$. Since $s_{i} \subseteq \mathrm{cl}_{X}{ }^{i} S_{0}$, it follows from Lemma 2.1 of [10] that there is an ultrafilter $G$ containing $S_{0}$ such that $F \geq \mathrm{cl}_{X}{ }^{i} G$. It is clear that $G$ cannot contain any set of the form $A_{j 0}$, and consequently $G \rightarrow x$ in $X$. Thus $F \rightarrow x$ in $r_{1} X$. If $H$ is an uitrafilter which contains no $S_{k}$ but has the property that each set $H \in H$ has an infinite intersection with infinitely many $S_{k}$ 's, then it follows from the preceding argument that $x^{\bullet} \geq \mathrm{cl}_{r_{1} X^{H}}$. Since $H \rightarrow z$ in $X$ it follows that $x^{\cdot} \rightarrow z$ in $r_{2} X$, and this leads to the result that $z^{\bullet}+x$ in $R_{3} X$. It remains only to show that $z^{\bullet}+x$ in $R_{2} X$. This follows from the fact that $z \notin \mathrm{cl}_{r_{1}} X^{n_{S}}$ for all $n \in N$, a result which can be easily established by induction on $n \cdot / /$ 


\section{Regular compactifications and completions}

Various investigations of compactifications of convergence spaces and completions of such related structures as uniform convergence spaces, convergence groups, and Cauchy spaces have consistently shown that regularity is an essential ingredient in obtaining extension theorems for canonical maps. (See, for instance, [6], [11], [13], [14], [15], and [17].) In this section we show that given any strict compactification $(Y, f)$ of a space $X$, the $R$-series can be employed to obtain a strict regular compactification $\left(y_{r}, f\right)$ of the regular modification $X_{r}$ of $X$. An analagous result is also obtained for completions of totally bounded convergence groups.

PROPOSITION 3.1. Let $f: X \rightarrow Y$ be a continuous function. Then $f: r_{\alpha} X \rightarrow r_{\alpha} Y$ is continuous for all $\alpha$. In particular, $f: X_{r} \rightarrow Y_{r}$ is continuous.

Proof. For $\alpha=0$, the result is given. Assume that $f$ is continuous for $\beta<\alpha$. If $F \rightarrow x$ in $r_{\alpha} X$, then $F \geq c l_{r_{\beta}}^{n_{G}}$, for some $n \in N, \beta<\alpha$, and $G \rightarrow x$ in $X$. Since $f: r_{\beta} X \rightarrow_{r_{\beta}}$ is continuous, $f\left(c l_{r_{B} X}{ }^{n} G\right) \geq \operatorname{cl}_{r_{B} Y}{ }^{n} f(G)$. Since $f(G) \rightarrow f(x)$ in $Y$, it follows that $f(F) \rightarrow f(x)$ in $r_{\alpha} Y$. //

A subset $B$ of a space $X$ is said to be strictly dense if ${ }^{c l_{X} B}=X$, and, whenever $F \rightarrow x$ in $X$, there is $G \rightarrow x$ such that $B \in G$ and $F \geq \mathrm{cl}_{X} G$. If $X$ is a topological space, then all dense subsets are strictly dense. A compactification $(Y, f)$ of a topological space is called strict if $f(X)$ is strictly dense in $Y$. The term strict completion (say, of a convergence group) is defined analagously. As far as we know, all compactifications and completions constructed to date in the literature have been strict, although they are not usually so designated. The significance of "strictness" in the study of regular completions and compactifications is pointed out in [11].

THEOREM 3.2. Let $(Y, f)$ be a strict compactification of $X$. Then $\left(r_{\alpha} Y, f\right)$ is a strict compactification of $r_{\alpha} X$ for all $\alpha$. In 
particular, $\left({ }_{r} f\right)$ is a strict compactification of $X_{r}$.

Proof. By Proposition 3.1, $f: r_{\alpha} X \rightarrow r_{\alpha} Y$ is continuous for all $\alpha$. Since the $r_{\alpha}^{Y^{\prime} \mathrm{s}}$ form a descending ordinal series, each $r_{\alpha}^{Y}$ is compact, and $f\left(r_{\alpha} X\right)$ is strictly dense in $r_{\alpha} Y$ for all $\alpha$. It remains only to show that $f^{-1}:\left.r_{\alpha} Y\right|_{\operatorname{Ran} f^{+}} r_{\alpha} X$ is continuous for all $\alpha$. Noting that for $\alpha=0$ the result is given, we proceed by induction on $\alpha$. Assume that $\left(r_{\beta} Y, f\right)$ is a strict compactification of $r_{\beta} X$ for $\beta<\alpha$. Let $G \rightarrow y$ in $r_{\alpha} Y$, where $G$ is a filter on $Y$ containing Ran $f$, and $y \in \operatorname{Ran} f$. Then $G \geq \mathrm{cl}_{r_{\beta} Y}{ }^{n} H$, for some $n \in N, \beta<\alpha$, and $H \rightarrow y$ in $Y$. By the strictness condition, there is a filter $F \rightarrow y$ in $Y$ such that $\operatorname{Ran} f \in F$ and $H \geq c l_{Y} F$. Thus $G \geq \mathrm{cl}_{r_{\beta} Y}{ }^{n}\left(c l_{Y} F\right) \geq \mathrm{cl}_{r_{B}}{ }^{n+l_{F}} F$. Since $f^{-1}:\left.r_{B} Y\right|_{\operatorname{Ran}} f^{\rightarrow} r_{B}^{X}$ is continuous,

$$
f^{-1}(G) \geq f^{-1}\left(c l_{r_{\beta} I}^{n+1} F\right) \geq c 1_{r_{\beta} X}^{n+1}\left(f^{-1}(F)\right),
$$

and $f^{-1}(F) \rightarrow f^{-1}(y)$ in $X$. Thus, $f^{-1}(G) \rightarrow f^{-1}(y)$ in $r_{\alpha} X$, and the proof is complete.

COROLLARY 3.3. If $X$ has a strict R-Hausdorff compactification, then $X_{r}$ has the same ultrafilter convergence as a Tychonoff topological space.

Proof. If $(Y, f)$ is a strict $R$-Hausdorff compactification of $X$, then $\left(Y_{p}, f\right)$ is a Hausdorff regular compactification of $X_{p}$. The assertion then follows immediately from Theorem 1, [16]. //

We use the term convergence group to mean an abelian group (using + as the operation, 0 as the identity element) equipped with a convergence structure which satisfies the following condition: $F \rightarrow x$ and $G \rightarrow y \Rightarrow F-G+x-y$.

THEOREM 3.4. If $X$ is a convergence group, then $r_{\alpha} X$ is a 
convergence group for all $\alpha$. In particular, $x_{r}$ is a regular convergence group.

Proof. Assume that $r_{\beta} X$ is a convergence group for $\beta<\alpha$. Let $F \rightarrow x$ and $G \rightarrow y$ in $r_{\alpha} X$. Then $F \geq{ }^{c l_{r_{\beta}}}{ }^{n_{H}}$ and $G \geq c^{c l_{r_{\gamma}}}{ }^{k} K$, for some $n$ and $k$ in $N, \beta$ and $\gamma$ less than $\alpha$, and $H \rightarrow x, K \rightarrow y$ in $X$. With no loss of generality, assume $\beta \geq \gamma$ and $n \geq k$. Then $F-G \geq c_{r_{\beta} X} n_{H}-c_{r_{\beta}}{ }^{n_{K}}{ }$. Using the hypothesis that ${ }_{r_{\beta} X}$ is a convergence group, we have $F-G \geq c_{r_{\beta} X}{ }^{n}(H-K)$, and $H-K \rightarrow x-y$. Thus we obtain $F-G \rightarrow x-y \cdot \quad / /$

The preceding theorem gives a method for constructing the regular convergence group which best approximates a given convergence group. It also leads to the following theorem concerning completions of totally bounded convergence groups.

A convergence group is totally bounded if each ultrafilter $F$ is Cauchy (that is, if $F-F \rightarrow 0$ in $X$ ), and complete if every Cauchy filter converges.

THEOREM 3.5. If $(Y, f)$ is a strict completion of a totally bounded convergence group $X$, then $\left(r_{\alpha}, f\right)$ is a strict completion of $r_{\alpha} X$ for alz $\alpha$. In particular, $\left(Y_{r}, f\right)$ is a strict regular completion of $X_{r}$.

Proof. By Theorem 3.4, $r_{\alpha} Y$ is a convergence group; it is clearly totally bounded, and therefore complete, since a cauchy filter $F$ is necessarily convergent if there is a convergent ultrafilter $G \geq F$. By Proposition 3.I, $r_{\alpha} Y$ is complete for all $\alpha$. It remains only to show that $f^{-1}$ is continuous, and this argument is identical to that given in the proof of Theorem 3.2. //

\section{Quotient maps}

In this section, we investigate the behavior of the $R$-series relative to certain types of quotient maps. Let $f: X \rightarrow Y$ be a map; that is, a 
continuous, onto function. If $Y$ has the finest convergence structure relative to which $f$ is continuous, then $f$ is called a convergence quotient map. It is noted in [9] that $f$ is a convergence quotient map iff, whenever $F+y$ in $Y$, there is $x \in f^{-1}(y)$ and $G \rightarrow x$ in $X$ such that $f(G)=F . f$ is said to be a proper map if, whenever $F$ is an ultrafilter on $Y, F \rightarrow y$ in $Y$, and $G$ is an ultrafilter on $X$ which maps on $F$, then there is $x \in f^{-1}(y)$ such that $G \rightarrow x$ in $X$. A proper convergence quotient map will be called a perfect map.

THEOREM 4.1. If $f: X+Y$ is a proper map, then $f: r_{\alpha} X \rightarrow r_{\alpha} Y$ is proper for all $\alpha$, and $\tau_{R} Y \leq \tau_{R} X+1$.

Proof. Assume that $f$ is proper for all ordinals $\beta<\alpha$. Let $F$ be an ultrafilter such that $F \rightarrow y$ in $r_{\alpha} Y$. Then there is $G \rightarrow y$ in $Y$ such that $F \geq{ }^{c}{ }_{r_{\beta}}{ }^{n} G$, for some $n \in N$ and $\beta<\alpha$. Using Lemma 1.1, we can assume without loss of generality that $G$ is an ultrafilter. Let $H$ be an ultrafilter on $X$ which maps on $F$. By the induction hypothesis, $f: r_{\beta} X \rightarrow r_{\beta} Y$ is proper, and it is shown in Proposition 3.2, [9], that proper maps preserve all iterations of the closure operator. Thus $H \geq f^{-1}\left(c_{r_{B}}{ }^{n} G\right)=c_{r_{B} X} f^{-1} G$. Applying Lemma 1.1 again, there is an ultrafilter $K \geq f^{-1} G$ such that $H \geq \mathrm{cl}_{r_{\beta}}{ }^{n} K$. Since $K$ is an ultrafilter which maps on $G$ and $f: X \rightarrow Y$ is proper, there is $x \in f^{-1}(y)$ such that $K \rightarrow x$ in $X$. Thus $H \rightarrow x$ in $r_{\alpha} X$, which proves that $f: r_{\alpha} X \rightarrow r_{\alpha}^{Y}$ is proper.

Finally, let $\gamma=z_{R} X$. Then $f: r_{\gamma} X \rightarrow r_{\gamma} Y$ is proper, and $r_{\gamma} X$ is regular. It is easy to check that $r_{\gamma} Y$ has the property described in Proposition 1.2 , and so $\rho\left(r_{\gamma} Y\right)$ is regular. Thus, by Proposition 2.4, $z_{R}\left(r_{\gamma} Y\right) \leq 1$, and it follows that $\tau_{R}(Y) \leq \gamma+1$. //

COROLLARY 4.2. The properties Urysohn and R-Hausdorff are preserved by proper maps. 
Proof. Let $f: X \rightarrow Y$ be a proper map. If $X$ is Urysohn, then $r_{1} X$ is Hausdorff, and $r_{1} Y$, being the image of a Hausdorff space under a proper map, is also Hausdorff (see [9]). Thus $Y$ is a Urysohn space. The same argument can be used if $X$ is R-Hausdorff, replacing $r_{1} X$ by $X_{r}$ and $r_{1} Y$ by $Y_{r}$. //

COROLLARY 4.3. If $f: X \rightarrow Y$ is proper and $X$ is R-Hausdorff, then $\tau_{R}(Y)$ and $\tau_{R}(X)$ differ by at most 1 ..

Proof. Let $\gamma=\tau_{R} Y$. By Proposition 2.2, it is sufficient to show that ${ }^{r} \gamma$ and $r_{\gamma+1} X$ have the same ultrafilter convergence. Let $F$ be an ultrafilter such that $F \rightarrow x$ in $r_{\gamma+1} X$, then $f(F) \rightarrow f(x)=y$ in $r_{\gamma+1} Y=r_{\gamma} Y$. Since $f: r_{\gamma} X \rightarrow r_{\gamma}^{Y}$ is a proper map, there is $z \in f^{-I}(y)$ such that $F \rightarrow z$ in $r_{\gamma} \dot{X}$. But $r_{\gamma+1} X$ is Hausdorff, and so $z=x \cdot / /$

The condition that $X$ is $R$-Hausdorff cannot be deleted in Corollary 4.3. For if $X$ is the space of Example 2.7, $Y$ the space consisting of a single point, and $f$ the constant map from $X$ onto $Y$, then $f$ is proper, but $\imath_{R} X \geq 3$, whereas $\imath_{R}^{Y}=0$.

THEOREM 4.4. If $f: X \rightarrow Y$ is a perfect mosp, then $f: r_{\alpha} X \rightarrow r_{\alpha} Y$ is perfect for all $\alpha$. In particular, $f: X_{r} \rightarrow y_{r}$ is perfect, and $\imath_{R} Y \leq \imath_{R} X$

Proof. Assume that $f$ is perfect for all $\beta<\alpha$. Then $f: r_{\alpha} X \rightarrow r_{\alpha} Y$ is proper by mheorem 4.1, and it remains only to show that it is a convergence quotient map. Let $F \rightarrow y$ in $r_{\alpha}{ }^{Y}$. Then there is $G \rightarrow y$ in $Y, n \in N$, and $\beta<\alpha$ such that $F \geq c{ }_{r_{\beta} Y}{ }_{G}$. Since $f: X \rightarrow Y$ is a convergence quotient map, there is $x \in f^{-1}(y)$ and $H \rightarrow x$ in $X$ such that $f(H)=G$. Since $f: r_{\beta} X \rightarrow r_{\beta} Y$ is proper, and thus closure-preserving, $f\left(\mathrm{cl}_{r_{\beta} X} n_{H}\right)=\mathrm{cl}_{r_{\beta} Y} n_{G}$. Let $k=f^{-1}(F) \vee c l_{r_{\beta} X} n_{H}$. Then $K \rightarrow x$ in $r_{\alpha} X$ and $f(K)=F$; therefore $f: r_{\alpha} X r_{\alpha} r_{\alpha}$ is a 
convergence quotient map.

It is easy to verify that regularity is preserved under perfect maps. Thus the image of $X_{p}$ under $f$ is regular, and consequently equal to $Y_{p}$. From this $Z_{R} Y \leq Z_{R}^{X}$ follows immediately. //

A continuous map $f: X \rightarrow Y$ is called a retraction if $Y$ is a subspace of $X$ and $f$ reduces to an identity map when restricted to $Y$.

THEOREM 4.5. If $f: X \rightarrow Y$ is a retraction, then $f: r_{\alpha} X \rightarrow r_{\alpha} Y$ is a retraction for alz $\alpha$. In particular, $f: X_{p} \rightarrow Y_{r}$ is a retraction, and $\tau_{R} y \leq \tau_{R} X$.

Proof. Assume that $f$ is a retraction for $\beta<\alpha$. We will first show that $r_{\alpha} Y$ is a subspace of $r_{\alpha} X$. Let $F \rightarrow y$ in ${ }^{r}{ }_{\alpha} Y$; then there are $n \in N, B<\alpha$, and $G \rightarrow y$ in $Y$ such that $F \geq c{ }_{r_{\beta} Y}{ }^{n}$. But

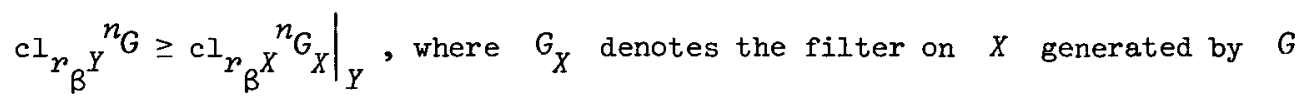
(which can be regarded as a filter base on $X$ ), and so $F \rightarrow y$ in $\left.r_{\alpha} X\right|_{Y}$. On the other hand, let $F \rightarrow y$ in $\left.r_{\alpha} X\right|_{Y}$. Then there are $n \in N$, $\beta<\alpha$, and $G \rightarrow y$ in $X$ such that $F_{X} \geq c 1_{r_{\beta} X} n_{G}$. Since $f: r_{\beta} X \rightarrow r_{\beta} Y$ is a retraction, $F=f\left(F_{X}\right) \geq f\left(c_{r_{B} X} n_{G}\right) \geq c_{r_{B}}{ }^{n} f(G)$. Also, $f(G) \rightarrow f(y)=y$ in $Y$, and so $\mathrm{cl}_{r_{\beta}}{ }^{n} f(G) \rightarrow y$ in $r_{\alpha}^{Y}$. Thus $r_{\alpha} Y=\left.r_{\alpha} X\right|_{Y}$

Proposition 3.1 establishes that $f: r_{\alpha} X r_{\alpha} Y$ is continuous, and hence a retraction. If $\gamma=Z_{R} X$, then $r_{\gamma} Y$ is a subspace of $r_{\gamma} X$ and is therefore regular. Thus $r_{\gamma} Y=y_{r}$, and so $z_{R} Y \leq z_{R} X$. //

Neither convergence quotient maps nor open maps (see [9]) are "wellbehaved" relative to the $R$-series. In the former case, it can be shown that each space is the image of a regular topological space under a convergence quotient map. There are also examples of spaces $X$ and $Y$ 
such that $f: X \rightarrow Y$ is an open map, but $f: r_{1} X \rightarrow r_{1} Y$ is not open.

\section{Subspaces}

Theorem 4.5 shows that certain types of subspaces, namely retracts, behave in a predictable fashion relative to the $R$-series. This section shows that the relationship between the $R$-series of a space and that of an arbitrary subspace tends to be rather erratic.

This first part of the proof of Theorem 4.5 can be applied to prove the following lemma.

LEMMA 5.1. If $X$ is a subspace of $Y$. then $r_{\alpha} X \geq\left. r_{\alpha} Y\right|_{X}$.

PROPOSITION 5.2. The R-Hausdorff property is hereditary.

Proof. Let $X$ be a subspace of an $R$-Hausdorff space $Y$, and let $\gamma=\imath_{R} X$. Then $X_{r}=r_{\gamma} X \geq\left. r_{\gamma} Y\right|_{X} \geq\left. Y_{r}\right|_{X}$, by Lemma 5.1. Since $Y_{r}$ is Hausdorff, $X_{p}$ must also be Hausdorff. //

THEOREM 5.3. Each Hausdorff space $X$ is a closed subspace of a Hausdorff space $Y$, where $l_{R}(Y)=1$.

Proof. Let $\alpha$ be the cardinal number of $X$; let $X=\left\{x_{\beta}: \beta \in I\right\}$, where $I$ is an index set of cardinality $\alpha$. Let $A=\left\{a_{B}: B \in I\right\}$ be a set of cardinality $\alpha$ which is disjoint from $X$, and let $Y=A \cup X$. Let $C=\{B \subseteq A$ : cardinality of $B$ is $\alpha\}$. For each $B \in C$, choose a collection $\left\{F_{B B}: \beta \in I\right\}$, where $F_{B B}$ is a free ultrafilter on $y, B \in F_{B \beta}$ for all $B \in I$, and $F_{B B}=F_{C \gamma}$ iff $B=C$ and $\beta=\gamma$. Let $x_{0}$ be an arbitrary point in $X$.

Next, we equip $X$ with the finest convergence structure which satisfies the following conditions:

(a) for each $B \in C$ and $B \in I, F_{B B} \rightarrow x_{B}$;

(b) for each free ultrafilter $G$ on $Y$ which contains $A$ and is not one of the $F_{B B}$ 's, $F \rightarrow x_{0}$;

(c) if $F$ is any filter on $Y$ which contains $X$, then $F \rightarrow x$ 
in. $Y$ iff $x \in X$ and $\left.F\right|_{X}+x$ in $X$.

To show that $Z_{R} Y=1$, we will show that $r_{I} Y$ is regular. First, we describe the $r_{1} Y$-convergent filters.

(i) If $F$ is a filter on $Y$ which contains $A$ and has the property that each $F \in F$ has cardinality $\alpha$, then $X \subseteq \mathrm{cl}_{Y} F$, for all $F \in F$, and hence $\mathrm{cl}_{Y} F=F \cap X^{\circ}$. Since no filters containing $X$ can $Y$-converge to any point of $A, c_{Y}{ }^{n} F=F \cap X^{*}$, for all $n \in N$. Each such filter $F \quad Y$-converges to a unique point $x \in X$, and it follows that $F r_{1} Y-$ converges only to $x$.

(ii) If $G$ is any filter containing $X$, then $G \geq \operatorname{cl}_{Y} F_{B B}=F_{B \beta} \cap X^{\bullet}$ for each $B \in C$ and $\beta \in I$. Since each point in $X$ has a filter of the form $F_{B B}$ converging to it, it follows that $G r_{1} Y$-converges to each point in $X$.

(iii) If $H$ is any free ultrafilter on $Y$ which contains a subset $B$ of $A$ of cardinality less than $\alpha$, then $H \cap x_{0} \geq c{ }_{X} H \geq H \cap X^{\bullet}$, and, because of $(i i), c^{2}{ }^{2} H=H \cap X^{\circ}$. It is easy to see that $H r_{1} Y_{-}$ converges only to $x_{0}$. For any fixed ultrafilter $a^{\bullet}$, for $a \in A$, it is clear that $a^{\cdot}$ converges only to $a$ in $r_{1} Y$.

For the filters $F, G, H$, and $a^{\bullet}$ discussed in (i), (ii), and (iii), it can be easily verified that ${ }^{c l_{r_{1}}} X^{F}=F \cap X^{*}, c_{r_{1}} X^{G}=X^{\circ}$,

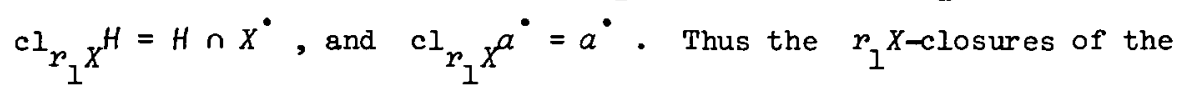
filters exhibit the same convergence in $r_{1} X$ as the filters themselves, and it follows that $r_{1} X$ is regular. //

If $X$ is the space of Example 2.7, and $Y$ is constructed as in Theorem 5.3, then $X$ is a closed subspace of $Y, Z_{R} X \geq 3$, and $\tau_{R} Y=1$.

COROLLARY 5.4. A closed subspace of a Hausdorff space can have a 
Zonger R-series than the space itself.

We conclude this section with two more examples. The first shows that a space $X_{1}$, with $Z_{R} X_{1} \geq 3$, can have a Hausdorff compactification $y_{0}$ such that $Z_{R} Y_{0}=2$. The second shows the reverse situation: a regular space $X_{0}$ which has a Hausdorff compactification $X$ such that $Z_{R} X \geq 3$.

EXAMPLE 5.5. Let $X_{0}$ be the subspace of the space $X$ constructed in Example 2.7 obtained by deleting from $X$ the point $\{y\}$. The reasoning of Example 2.7 shows that $\tau_{R} x_{0} \geq 3$.

Letting $X_{0}$ play the role of $X$ in Theorem 5.3, construct $Y$ as described in the proof of that theorem. Let $A=\left\{a_{n}: n \in N, n \geq 1\right\}$. Define $y_{0}$ to be the space with the same underlying set as $Y$, and equipped with the finest convergence structure which satisfies the following conditions:

(a) if $F \rightarrow y$ in $Y$, then $F \rightarrow y$ in $Y_{0}$;

(b) if $F$ is a free ultrafilter on $Y$ which contains $S_{n}$, for $n \geq 1$ (see Example 2.7), and $\left.F\right|_{X} \rightarrow y$ in $X$, then $F \rightarrow a_{n}$ in $Y_{0}$.

It is not difficult to show that $r_{2} Y_{0}$ is the indiscrete space, and so $z_{R} Y_{0} \leq 2<z_{R} X$. It is easy to see from the above construction that $Y_{0}$, along with the identity embedding, is a Hausdorff compactification of $X_{0}$

EXAMPLE 5.6. Let $X_{1}$ be the subspace obtained from the space $X$ of Example 2.7 by deleting the elements $x, y$, and $z$ from $X$. Then $X_{1}$ is a regular, Hausdorff space $\left(\tau_{R} X_{1}=0\right)$, and $X$ is a Hausdorff compactification of $X$ with $\tau_{R} X \geq 3$. // 


\section{Products and disjoint sums}

Let $\left\{x_{i}: i \in I\right\}$ be a family of spaces over an arbitrary index set $I$. The product space, with the product convergence structure, is denoted by $X ; X=\prod\left\{x_{i}: i \in I\right\}$. Let $P_{i}: X \rightarrow x_{i}$ be the $i$-th projection màp; the product convergence structure (that is, the structure of pointwise convergence) is the coarsest on $X$ relative to which all of the projection maps are continuous.

PROPOSITION 6.1. Let $X=\prod\left\{\left\{X_{i}: i \in I\right\}\right.$. Then, for any ordinal number $\alpha, \quad r_{\alpha} X \geq \prod\left\{r_{\alpha} X_{i}: i \in I\right\}$. Furthermore, $\tau_{R} X \geq \tau_{R} X_{i}$, for alz $i \in I$.

Proof. Assume that $r_{\beta} X \geq \prod\left\{r_{\beta} X_{i}\right\}$ for all $\beta<\alpha$. Let $F \rightarrow\left(x_{i}\right)$ in $r_{\alpha} X$. Then there are $n \in N, \gamma<\alpha$, and $G \rightarrow\left(x_{i}\right)$ in $X$ such that $F \geq{ }^{c l_{r_{\gamma} X}^{n_{G}}}$. Thus $P_{i} F \geq{ }^{c l_{r_{\gamma} X_{i}}}{ }_{P_{i} G}$, and so $P_{i} F \rightarrow x_{i}$ in $r_{\alpha}{ }^{X}$, for all $i \in I$. Therefore $F \rightarrow\left(x_{i}\right)$ in $\prod\left\{r_{\beta} X_{i}\right\}$. The last assertion follows from Theorem 4.5, since $P_{i}: X \rightarrow X_{i}$ is a retraction for each $i \in I . \quad / 1$

If each $x_{i}$ is $R$-Hausdorff, then $\prod\left\{\left(x_{i}\right)_{r}: i \in I\right\}$ is Hausdorff, and it follows from Proposition 6.1 that $X_{r}$ is Hausdorff. Thus we have

COROLLARY 6.2. A product of R-Hausdorff spaces is R-Hausdorff.

THEOREM 6.3. Let $X=\prod\left\{X_{i}: i \in I\right\}$, where $I$ is a finite index set. Then $r_{\alpha} X=\prod T\left\{r_{\alpha} X_{i}: i \in I\right\}$. In particular, $x_{r}=\prod\left\{\left(X_{i}\right)_{r}: i \in I\right\}$, and $z_{R} X=\sup \left\{z_{R} X: i \in I\right\}$.

Proof. Assume $r_{\beta} X=\prod\left\{r_{\beta} X_{i}: i \in I\right\}$ is valid for $\beta<\alpha$. Let $F \rightarrow\left(x_{i}\right)$ in $\prod\left\{r_{\alpha} X_{i}\right\}$. Then, for each $i \in I$, there are $n_{i} \in N$, $\beta_{i}<\alpha, G_{i}+x_{i}$ in $X_{i}$ such that $P_{i} F \geq{ }^{c l}{ }_{r_{B}} X_{i}{ }^{n}{ }_{G}$. Let $n=\sup \left\{n_{i}: i \in I\right\}, \beta=\sup \left\{B_{i}: i \in I\right\}$, and $G=\prod\left\{G_{i}: i \in I\right\}$. 
Then $F \geq \operatorname{cl}_{r_{B} X} n_{G}$, where $G \rightarrow\left(x_{i}\right)$ in $X$, and so $F \rightarrow\left(x_{i}\right)$ in $r_{\alpha} X$. This result, along with Proposition 6.1, establishes the first equality; the second is immediate. The third equality follows from Proposition 6.1 and the fact that a product of regular spaces is regular. //

A space $X$ is the disjoint sum of the family $\left\{X_{i}: i \in I\right\}$ if:

(1) $X$ is the union of the $x_{i}^{\prime}$ 's;

(2) the $X_{i}$ 's are pairwise disjoint;

(3) $F \rightarrow x$ in $X$ iff $F$ contains the set $X_{i}$ which contains $x$, and $\left.F\right|_{X_{i}} \rightarrow x$ in $X_{i}$.

We omit the straightforward proof of the next proposition.

PROPOSITION 6.4. If $X$ is the disjoint sum of $\left\{x_{i}: i \in I\right\}$, then $r_{\alpha} X$ is the disjoint sum of $\left\{r_{\alpha} X_{i}: i \in I\right\}$ for all ordinals $\alpha$, and $z_{R} X=\sup \left\{z_{R^{X}}{ }_{i}: i \in I\right\}$. //

\section{First countable spaces}

A convergence space $X$ is said to be first countable if, whenever $F \rightarrow x$, there is a filter $G \rightarrow x$ such that $G$ has a countable filter base and $F \geq G$.

PROPOSITION 7.1. If $X$ is a first countable space, then $r_{\alpha} X$ is first countable for all ordinal numbers $\alpha$.

Proof. Assume that the proposition is valid for $\beta<\alpha$. Let $F \rightarrow x$ in $X$ such that $F \geq \mathrm{cl}_{r_{\beta} X}{ }^{n} G$. Since $X$ is first countable, we can assume that $G$ has a countable filter base. Thus ${ }^{c l_{r_{B}}{ }^{n} G}$ is a filter which has a countable filter base, $r_{\alpha} X$-converges to $x$, and is coarser than $F$. Thus $r_{\alpha} X$ is first countable. //

For any space $X$, let $x^{0}$ be the space with the same underlying set, 
and with convergence defined as follows: $F \rightarrow x$ in $X^{0}$ iff $F \rightarrow x$ in $X$ and there is a filter $G$ with a countable base such that $G \rightarrow x$ in $X$ and $F \geq G . X^{0}$ is the coarest first countable space finer than $X$, and is called the first countable modification of $X$.

PROPOSITION 7.2. If $X$ is a regular space, then $X^{0}$ is regular.

Proof. Let $F \rightarrow x$ in $X^{0}$, and let $G$ be a countable base filter such that $F \geq G$ and $G \rightarrow x$ in $X$. Then $\mathrm{cl}_{X^{0}} F \geq \mathrm{cl}_{X} G$, and $\mathrm{cl}_{X} G$ is a countable base filter which converges to $x$ in $X$, since $X$ is regular. //

It is obvious that $\left(X_{p}\right)^{0} \leq\left(X^{0}\right)_{p}$ for any space $X$. If $X$ is an uncountable set with the cofinite topology, then $\left(x_{p}\right)^{0}$ is indiscrete, whereas $\left(X^{0}\right)_{r}$ is discrete.

We shall next show that, for any first countable space $X$, $z_{R} X \leq \omega_{1}+1$, where $\omega_{1}$ is the first uncountable ordinal.

LEMMA 7.3. Let $X$ be a first corntable space, and let $\left\{A_{\sigma}: 0 \leq \sigma<\omega_{1}\right\}$ be a collection of subsets of $X$ such that $A_{\alpha} \subseteq A_{\beta}$ for $0 \leq \alpha \leq \beta<\omega_{1}$. Then

$$
{ }^{c I_{\omega_{1}} X}{ }^{n}\left(U\left\{A_{\sigma}: \sigma<\omega_{1}\right\}\right)=U\left\{c{ }_{r_{\sigma} X}^{n} A_{\sigma}: \sigma<\omega_{1}\right\} \text {. }
$$

Proof. Consider the case $n=1$. Let $x \in \mathrm{cl}_{r_{\omega_{1}} X} \mathrm{U}\left(A_{\sigma}\right)$; then there is $\gamma<\omega_{1}$ and $F \rightarrow x$ in $X$ such that $F$ has a countable base $\left\{F_{n}\right\}$ and $\left(\mathrm{cl}_{r_{\gamma} X}{ }^{n_{F}}\right) \vee\left(U\left\{A_{\beta}: \beta<\omega_{1}\right\}\right) \neq \emptyset^{\circ}$. For each $k \geq 1$, there is $B_{k}<\omega_{1}$ such that $\left({ }^{c}{ }_{r_{\gamma} X}{ }^{n} F_{k}\right) \cap A_{B_{k}} \neq \emptyset$. Let $\alpha=\sup \left\{\beta_{k}: k \in N\right\}$. Then $\alpha<\omega_{1}$, and $\operatorname{cl}_{r_{\gamma} X} n_{F} \vee A_{\alpha} \neq \phi^{\circ}$. Let $\delta$ be the larger of the 
ordinals $\gamma$ and $\alpha$; then ${ }^{c I_{r_{\delta}}{ }^{n} F \vee A_{\delta}} \neq \emptyset^{\circ}$, and so $x \in{ }^{c 1} r_{\delta+1} X^{A} \delta+1$ Thus ${ }^{c l_{\omega_{1}} X}\left(U A_{\sigma}\right) \subseteq U\left(c l_{r_{\sigma} X} n_{A_{\sigma}}\right)$. Inclusion in the other direction is clear.

Finally, assume that the assertion of the lema is valid for $k$. Then $c l_{r_{\omega_{1}} X}{ }^{k+1}\left(U A_{\sigma}\right)=\mathrm{cl}_{r_{\omega_{1}} X}\left(\mathrm{cl}_{r_{\omega_{1}} X}{ }^{k}\left(U A_{\sigma}\right)\right)=\mathrm{cl}_{r_{\omega_{1}} X}\left(U\left(c l_{r_{\sigma} X}{ }^{k} A_{\sigma}\right)\right)$. Since the sets ${ }^{c l_{r_{\sigma} X}}{ }^{k_{A}}$ satisfy the conditions assumed in the lemma we have ${ }^{c l_{\omega_{1}} X}{ }^{k+1}\left(U A_{\sigma}\right)=U\left(\mathrm{cl}_{r_{\sigma}}{ }^{k+1} A_{\sigma}\right)$, and the proof is complete. /1

THEOREM 7.4. If $X$ is a first countable space, then $z_{R} X \leq \omega_{1}+1$.

Proof. By Proposition 2.2, it suffices to show that ${ }^{c l_{\omega_{1}+1}} X^{A={ }^{c l} r_{\omega_{1}}} X^{A}$. Let $x \in{ }^{c l} r_{\omega_{1}+1} X^{A} ;$ then there is $F \rightarrow x$ in $X$ with a countable filter base $\left\{F_{n}\right\}$ such that $\left({ }^{c l_{\omega_{1}}}{ }^{n} F\right) \vee A^{\bullet} \neq \emptyset^{\circ}$. For each $k \geq 1, \quad\left(\operatorname{cl}_{c_{\omega_{1}} X} n_{F_{k}}\right) \cap A \neq \emptyset$. By Lemma $7 \cdot 3$, there is for each $k$, $\sigma_{k}<\omega_{1}$ such that $\left(\operatorname{cl}_{r_{\sigma_{k}}}{ }^{n} F_{k}\right) \cap A \neq \emptyset$. Let $\delta=\sup \left\{\sigma_{k}\right\} ;$ then $\left(c I_{r_{\delta} X} n_{F}\right) \vee A^{\bullet} \neq \phi^{\bullet}$, and so $x \in c^{c r_{\delta+1}} X^{A}$. Consequently

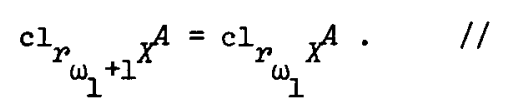

A space $X$ is said to have the countable intersection property if, whenever $F \rightarrow x$, there is a filter $G \rightarrow x$ such that $F \geq G$ and $G$ has a filter base which is closed under countable intersections. The next theorem can be established using arguments similar to those of the preceding theorem; we omit the details.

THEOREM 7.5. If a space $X$ has the countable intersection property, 
then $\tau_{R^{X}} \leq \omega+1$ (where $\omega$ denotes the first infinite ordinal).

\section{The D-series}

The decomposition series $\left\{\pi_{\alpha} X\right\}$ of a convergence space $X$ was defined in [10] to be the ordinal series of pretopologies corresponding to the distinct iterations of the closure operator of $X$. The term "decomposition series" will be shortened to D-series. The length of this series, which we will denote here by $l_{D}(X)$, is the smallest ordinal $\gamma$ such that $\mathrm{cl}_{X}^{\gamma_{A}}=\mathrm{cl}_{X}{ }^{\gamma+1} A$, for all $A \subseteq X$. The $D$-series terminates in the topological modification $\lambda X$ of $X$.

A non-regular topological space has a D-series of length 0 , but the length of its $R$-series must be at least 1 . The spaces $(S, r)$ and $(S, q)$ of Example 2.10, [10], have infinitely long $D$-series; however these spaces are regular, and hence have $R$-series of length 0 . It is not easy to find relationships between $Z_{D}(X)$ and $Z_{R}(X)$; however the next proposition establishes such a relationship for a certain class of spaces.

PROPOSITION 8.1. If $X$ is a space such that $X_{r} \geq \lambda X$ and $\tau_{R}(X)>1$, then $\tau_{D}(X)$ is infinite.

Proof. Assume that $Z_{D}(X)=n<\omega$. Then, for each filter $G$ on $X, \mathrm{cl}_{X}^{n_{G}}=\mathrm{cl}_{\lambda X} G$. Thus $F \rightarrow x$ in $r_{I} X$ iff there is $G \rightarrow x$ in $X$ such that $F \geq{ }^{c l_{\lambda X}}{ }^{G}$. Since $r_{1} X \geq \lambda X$ by assumption, ${ }^{c l_{r_{1}}}{ }^{n_{G}}={ }^{c l}{ }_{\lambda X}{ }^{G}$ is also valid for all filters $G$ on $X$. By the definition of $r_{2} X$, it follows that $r_{1} X=r_{2} X$, and consequently $Z_{R}(X)=1$, a contradiction. //

We conclude by making some comparisons between the $R$-series and $D$-series. There is no predictable relationship, in general, between the length of the $D$-series of a subspace and that of the original space; however, if the subspace is either open or closed, the length of the $D$-series of the subspace cannot exceed that of the original space. In the case of the $R$-series, the unpredictable behavior extends even to closed 
and open subspaces. The length of the $D$-series for a disjoint sum of spaces is the supremum of the lengths of the $D$-series for the component spaces; the same is true for the $R$-series. The $R$-series is well behaved relative to finite products; the $D$-series is not. The upper bounds established in Section 7 for the $R$-series of first countable spaces and spaces with the countable intersection property are essentially the same as those obtained for the $D$-series for the same classes of spaces in [10].

\section{References}

[1] H.J. Biesterfeldt, Jr., "Regular convergence spaces", K. NederZ. Akad. Wetensch. Proc. Ser. A 69 = Indag. Math. 28 (1966), 605-607.

[2] Eduard Čch, Topological spaces, revised ed. (Publishing House of the Czechoslovak Academy of Sciences, Prague; Interscience [John Wiley \& Sons], London, New York, Sydney; 1966).

[3] C.H. Cook and H.R. Fischer, "Regular convergence spaces", Math. Ann. 174 (1967), 1-7.

[4] H.R. Fischer, "Limesräume", Math. Ann. 137 (1959), 269-303.

[5] R. Frič, "Regularity and extension of mappings in sequential spaces", Comment. Math. Univ. Carolinae 15 (1974), 161-171.

[6] R.J. Gazik and D.C. Kent, "Regular completions of Cauchy spaces via function algebras", Bull. Austral. Math. Soc. 11 (1974), 77-88.

[7] Brian V. Hearsey, "Regularity in convergence spaces", Portugal Math. 30 (1971), 201-213.

[8] D.C. Kent and G.D. Richardson, "Minimal convergence spaces", Trans. Amer. Math. Soc. 160 (1971), 487-499.

[9] Darrell C. Kent and Gary D. Richardson, "Open and proper maps between convergence spaces", Czechoslovak Math. J. 23 (98) (1973), $15-23$.

[10] Darrell C. Kent and Gary D. Richardson, "The decomposition series of a convergence space", Caechoslovak Math. J. 23 (98) (1973), $437-446$.

[11] D.C. Kent and G.D. Richardson, "Regular completions of Cauchy spaces", Pacific J. Math. 51 (1974), 483-490. 
[12] Josef Novák, "On convergence spaces and their sequential envelopes", Czechoslovak Math. J. 15 (90) (1965), 74-100.

[13] Ellen E. Reed, "Completions of uniform convergence spaces", Math. Ann. 194 (1971), 83-108.

[14] G.D. Richardson, "A stone-Čech compactification for limit spaces", Proc. Amer. Math. Soc. 25 (1970), 403-404.

[15] G.D. Richardson, "Completions for a class of convergence groups", Proc. Amer. Math. Soc. 39 (1973), 211-213.

[16] G.D. Richardson and D.C. Kent, "Regular compactifications of convergence spaces", Proc. Amer. Math. Soc. 31 (1972), 571-573.

[17] Oswald Wyler, "Ein Komplettierungsfunktor für uniforme Limesräume", Math. Nachr. 46 (1970), 1-20.

Department of Mathematics,

East Carolina University,

Greenville,

North Carol ina,

USA ;

Department of Pure and Appl ied Mathematics, Washington State University,

Pul Iman,

Washington,

USA. 